PROCEEDINGS OF THE AMERICAN MATHEMATICAL SOCIETY

Volume 124, Number 9, September 1996

\title{
LINDELÖF POWERS AND PRODUCTS OF FUNCTION SPACES
}

\author{
OLEG OKUNEV AND KENICHI TAMANO \\ (Communicated by Franklin D. Tall)
}

\begin{abstract}
We give criteria for finite and countable powers of a space similar to the Michael line being Lindelöf. As applications, we give examples related to Lindelöf property in products of spaces of Michael line type and in products of spaces of continuous functions on separable $\sigma$-compact spaces.
\end{abstract}

All spaces considered below are assumed to be Tychonoff (= completely regular Hausdorff). We denote by $C_{p}(X)$ the space of all continuous real-valued functions endowed with the topology of pointwise convergence on $X$; this topology can be obtained as the restriction of the Tychonoff product topology on the set $\mathbb{R}^{X}$ of all real-valued functions on $X$ to its subset $C(X)$ (see [Arh1]). $C_{p}(X, 2)$ is the subspace of $C_{p}(X)$ consisting of all functions to $2=\{0,1\}$. The symbols $\omega, \mathbb{R}, I$ and $\mathbf{C}$ stand for the set of naturals, the real line, the segment $[0,1]$, and the Cantor cube $2^{\omega}$. If $P$ and $Q$ are sets, then $P^{Q}$ denotes the set of all functions from $Q$ to $P$; if $\kappa$ is a cardinal, then $X^{\kappa}$ is the $\kappa$ th power of $X$ (with the Tychonoff product topology); the projection of $X^{\kappa}$ to its $i$ th factor is denoted by $\pi_{i}$. For $j \in 2$ and $\sigma \in 2^{i}$, denote $\sigma^{\frown} j=\sigma \cup\{\langle i, j\rangle\} \in 2^{i+1}$. The symbol $\mathfrak{c}$ denotes the cardinality of the continuum. Polish spaces are separable completely metrizable spaces.

\section{Holding SETS}

We study the following construction introduced by Bing and Hanner (see [Eng, 5.1.22]). Given a topological space $X$ and a subset $A$ of $X$, let $X_{(A)}$ be the space obtained by retaining the topology at each point of $A$ and by declaring the points of $X \backslash A$ isolated. Given two subsets $A$ and $B$ of $X$, we have a natural one-to-one mapping $i_{A B}: X_{(A)} \rightarrow X_{(B)}$ (the identity mapping of the underlying set $X$ ); clearly, $i_{A B}$ is continuous if $A \subset B$ (only if, if the space $X$ has no isolated points). Another simple observation is that the diagonal in $X_{(A)} \times X_{(B)}$ is closed and homeomorphic to $X_{(A \cap B)}$; in particular, if $A \cap B=\emptyset$, then the diagonal is closed and discrete. Furthermore, if $\mathcal{A}$ is a family of subsets of $X$, then the diagonal of the product $\prod\left\{X_{(A)}: A \in \mathcal{A}\right\}$ is closed and is homeomorphic to $X_{(\cap \mathcal{A})}$.

Call a subset $A$ of a space $X$ holding if the space $\left(X_{(A)}\right)^{\omega}$ is Lindelöf. The existence of a holding subset of $I$ with $|I \backslash A|=\mathfrak{c}$ was shown in [OY] under Martin's Axiom.

(1.1) Theorem. Let $X$ be a Polish space, and $A$ a subset of $X$. Then the following conditions are equivalent:

Received by the editors July 22, 1994 and, in revised form, March 2, 1995.

1991 Mathematics Subject Classification. Primary 54C35, 54D20.

Key words and phrases. Sets of reals, products, Lindelöf spaces, function spaces. 
(1) $A$ is holding,

(2) $\bigcap\left\{f^{-1}(A): f \in \Psi\right\} \neq \emptyset$ for any countable family $\Psi$ of finite-to-one continuous mappings of the Cantor cube $\mathbf{C}=2^{\omega}$ to $X$.

Proof. $(1) \Rightarrow(2)$. Suppose there is a countable family $\left\{f_{n}: n \in \omega\right\}$ of finite-to-one continuous mappings from $\mathbf{C}$ to $X$ such that $\bigcap\left\{f_{n}^{-1}(A): n \in \omega\right\}=\emptyset$. For each $n \in \omega$, put $B_{n}=f_{n}^{-1}(A), Y_{n}=\mathbf{C}_{\left(B_{n}\right)}$ and consider the mapping $g_{n}: Y_{n} \rightarrow X_{(A)}$ that acts the same way as $f_{n}$ (so formally, $g_{n}=i_{A X}^{-1} \circ f_{n} \circ i_{B_{n} \mathbf{C}}$ ). Clearly, $g_{n}$ is continuous. Furthermore, $g_{n}$ is closed. Indeed, let $F$ be a closed set in $\mathbf{C}_{\left(B_{n}\right)}$; then it is of the form $P \backslash L$ where $P$ is closed in $\mathbf{C}$ and $L \subset \mathbf{C} \backslash B_{n}$. It follows that $f_{n}(P) \cap A \subset g_{n}(F) \subset f_{n}(P)$, and since $f_{n}(P)$ is closed in $X, g_{n}(F)$ is closed in $X_{(A)}$. Since $g_{n}$ is also finite-to-one, it is perfect. Therefore, the product mapping $g=\prod g_{n}: \prod\left\{Y_{n}: n \in \omega\right\} \rightarrow\left(X_{(A)}\right)^{\omega}$ is perfect (see [Eng, 3.7.6]).

Since $\bigcap\left\{B_{n}: n \in \omega\right\}=\bigcap\left\{f_{n}^{-1}(A): n \in \omega\right\}=\emptyset$, the diagonal $\Delta$ is closed and discrete in $\prod\left\{Y_{n}: n \in \omega\right\}$, so its perfect image $g(\Delta)$ is a closed, uncountable discrete subspace of $\left(X_{(A)}\right)^{\omega}$. Thus, $\left(X_{(A)}\right)^{\omega}$ is not Lindelöf, and the proof is complete.

$(2) \Rightarrow(1)$. We need two lemmas for this proof. The following lemma is proved by an obvious induction on $i$.

(1.2) Lemma. Let $X$ be a separable metric space and $Z$ an uncountable subset of $X^{n}$ such that $\pi_{i}(z) \neq \pi_{i}\left(z^{\prime}\right)$ for any distinct $z, z^{\prime} \in Z$ and $i<n$. Then for any $\epsilon>0$ there are subsets $Z_{0}$ and $Z_{1}$ of $Z$ such that

(i) $Z_{j}$ is uncountable,

(ii) $\operatorname{diam}\left(\pi_{i}\left(Z_{j}\right)\right) \leq \epsilon$, and

(iii) $\operatorname{cl}\left(\pi_{i}\left(Z_{0}\right)\right) \cap \operatorname{cl}\left(\pi_{i}\left(Z_{1}\right)\right)=\emptyset$

for all $i<n$ and $j \in 2$.

Let $S=\bigcup\left\{2^{n}: n \in \omega\right\}$. Denote $2^{\leq n}=\bigcup\left\{2^{k}: k \leq n\right\}$.

(1.3) Lemma. Let $X$ be a separable metric space. Suppose $F$ is an uncountable subset of $X^{\omega}$. Then there are a family $\left\{F_{\sigma}: \sigma \in S\right\}$ of uncountable subsets of $F$ and a function $\phi: S \rightarrow 2=\{0,1\}$ satisfying the following conditions:

(i) $F_{\sigma} \subset F_{\tau}$ for any $\sigma, \tau \in S$ with $\tau \subset \sigma$;

(ii) If $\phi(\sigma \mid i)=0$, then $\pi_{i}(y)=\pi_{i}\left(y^{\prime}\right)$ for any $\sigma \in S, y, y^{\prime} \in F_{\sigma}$ and $i \leq|\sigma|$;

(iii) If $\phi(\sigma \mid i)=1$, then $\pi_{i}(y) \neq \pi_{i}\left(y^{\prime}\right)$ for any $\sigma \in S$, distinct $y, y^{\prime} \in F_{\sigma}$ and $i \leq|\sigma|$

(iv) $\operatorname{diam}\left(\pi_{i}\left(F_{\sigma}\right)\right) \leq 1 / 2^{|\sigma|}$ for any $\sigma \in S$ and $i<|\sigma|$; and

(v) $\operatorname{cl}_{X}\left(\pi_{i}\left(F_{\sigma \frown 0}\right)\right) \cap \operatorname{cl}_{X}\left(\pi_{i}\left(F_{\sigma \frown 1}\right)\right)=\emptyset$ if $\sigma \in S, i \leq|\sigma|$ and $\phi(\sigma \mid i)=1$.

Proof of Lemma 1.3. We will define $\left\{F_{\sigma}: \sigma \in 2^{n}\right\}$ and $\phi \mid 2^{n}: 2^{n} \rightarrow 2$ by induction on $n$.

Let us first define $F_{\emptyset}$ and $\phi(\emptyset)$. If $\pi_{0}(F)$ is uncountable, then take an uncountable subset $F_{\emptyset}$ of $F$ such that $\pi_{0}(y) \neq \pi_{0}\left(y^{\prime}\right)$ for any distinct $y, y^{\prime} \in F_{\emptyset}$, and put $\phi(\emptyset)=1$. Otherwise, take an uncountable subset $F_{\emptyset}$ of $F$ such that $\pi_{0}(y)=\pi_{0}\left(y^{\prime}\right)$ for all $y, y^{\prime} \in F_{\emptyset}$ and put $\phi(\emptyset)=0$.

Assume that $\left\{F_{\sigma}: \sigma \in 2^{\leq n}\right\}$ and $\phi \mid 2^{\leq n}$ are already defined so that they satisfy (i)-(v) for all $\sigma, \tau \in 2^{\leq n}$. Let $\sigma \in 2^{n}$ and $j \in 2$; we need to define $F_{\sigma \wedge j}$ and $\phi\left(\sigma^{\frown} j\right)$. By (iv) of our inductive assumption and Lemma 1.2, we can take uncountable subsets $Z_{0}$ and $Z_{1}$ in $F_{\sigma}$ so that the diameters of $\pi_{i}\left(Z_{0}\right)$ and $\pi_{i}\left(Z_{1}\right)$ do not exceed $1 / 2^{n+1}$ and $\operatorname{cl}\left(\pi_{i}\left(Z_{0}\right)\right) \cap \operatorname{cl}\left(\pi_{i}\left(Z_{1}\right)\right)=\emptyset$ for all $i \leq n$ with $\phi(\sigma \mid i)=1$. If $\pi_{n+1}\left(Z_{j}\right)$ 
is uncountable, then let $F_{\sigma \sim j}$ be an uncountable subset of $Z_{j}$ such that $\pi_{n+1}(y) \neq$ $\pi_{n+1}\left(y^{\prime}\right)$ whenever $y \neq y^{\prime}, y, y^{\prime} \in F_{\sigma \frown j}$ and put $\phi\left(\sigma^{\wedge} j\right)=1$. Otherwise, let $F_{\sigma \frown j}$ be an uncountable subset of $Z_{j}$ such that $\pi_{n+1}(y)=\pi_{n+1}\left(y^{\prime}\right)$ for all $y, y^{\prime} \in F_{\sigma \sim j}$, and put $\phi\left(\sigma^{\wedge} j\right)=0$. Now it is easy to check that $\left\{F_{\sigma}: \sigma \in 2^{n+1}\right\}$ and $\phi \mid 2^{n+1}$ satisfy the desired conditions.

Let us now return to the proof of the theorem. Suppose (2) holds, but $A$ is not a holding set. Since $X_{(A)}$ is an Eberlein-Grothendieck space (see [Ok1, 1.111.12]), and the class of Eberlein-Grothendieck spaces is countably productive [Arh1], $Y=\left(X_{(A)}\right)^{\omega}$ is an Eberlein-Grothendieck space. By the Baturov's theorem [Bat], the Lindelöf number of $Y$ coincides with the extent. Since $Y$ is not Lindelöf, there is an uncountable discrete set $F$ in $Y$.

Note that for some $i \in \omega$, the projection $\pi_{i}(F)$ is uncountable, because $F$ is an uncountable closed discrete set in the product $\prod\left\{\pi_{i}(F): i \in \omega\right\}$. We assume without loss of generality that $\pi_{0}(F)$ is uncountable and $\pi_{0}(y) \neq \pi_{0}\left(y^{\prime}\right)$ whenever $y, y^{\prime} \in F, y \neq y^{\prime}$.

Fix a complete metric on $X$, and let $F_{\sigma}, \sigma \in S$ and $\phi: S \rightarrow 2$ be as in Lemma 1.3. By the above note and the construction of $\phi$, we have $\phi(\emptyset)=1$. For each $f \in 2^{\omega}$, $\bigcap\left\{\operatorname{cl}_{X^{\omega}}\left(F_{f \mid n}\right): n \in \omega\right\}$ is a singleton; this follows from conditions (i) and (iv) in Lemma 1.3 and the completeness of $X$. Define $y_{f}$ by

$$
\left\{y_{f}\right\}=\bigcap\left\{\operatorname{cl}_{X^{\omega}}\left(F_{f \mid n}\right): n \in \omega\right\} .
$$

Suppose that $i \in \omega, \sigma \in 2^{i}$ and $\phi(\sigma)=1$. For each $f \in 2^{\omega}$ with $f \supset \sigma$, put $\psi_{i \sigma}(f)=y_{f}(i)$. By the conditions (iv) and (v) of Lemma 1.3, $\psi_{i \sigma}$ is a one-to-one continuous mapping from $C_{\sigma}=\left\{f \in 2^{\omega}: \sigma \subset f\right\}$ to $X$. Put $C_{i}=\bigcup\left\{C_{\sigma}: \sigma \in\right.$ $\left.2^{i}, \phi(\sigma)=1\right\}$ and define a function $\psi_{i}: C_{i} \rightarrow X$ by $\psi_{i} \mid C_{\sigma}=\psi_{i \sigma}$ for all $\sigma \in 2^{i}$ with $\phi(\sigma)=1$. Take a finite-to-one continuous retraction $r$ from $2^{\omega}$ to its clopen subset $C_{i}$ and put $\psi_{i}^{\prime}=\psi_{i} \circ r$.

By the assumption (2), there is a function

$$
f \in \bigcap\left\{\psi_{i}^{\prime-1}(A): i \in \omega, \quad \phi(\sigma)=1 \quad \text { for some } \sigma \in 2^{i}\right\}
$$

(the family intersected in the right-hand side here is nonempty, because $\phi(\emptyset)=1$ ).

Let us show that $y_{f}$ is a limit point for $F$ in $\left(X_{(A)}\right)^{\omega}$, in contradiction with $F$ being closed discrete. Let $U=\bigcap\left\{\pi_{i}^{-1}\left(U_{i}\right): i<n\right\}$ be a basic neighborhood of $y_{f}$ in $\left(X_{(A)}\right)^{\omega}$. Take $k \in \omega$ such that if $x \in X, i<n,\left|\pi_{i}\left(y_{f}\right)-x\right| \leq 1 / 2^{k}$ and $U_{i}$ is a neighborhood of $y_{f}(i)$ in $X$, then $x \in U_{i}$. Put $m=\max \{k, n\}$ and $\sigma=f \mid m$. Let $i<n$; two cases are possible.

Case 1. $\phi(f \mid i)=1$.

Let $\tau=f \mid i$. Since $f \in \psi_{i}^{\prime-1}(A) \cap C_{\tau}=\psi_{i \tau}{ }^{-1}(A)$, we have $\psi_{i \tau}(f)=\pi_{i}\left(y_{f}\right) \in$ A. Therefore, $U_{i}$ is a neighborhood of $\pi_{i}\left(y_{f}\right)$ in $X$. By (iv) in Lemma 1.3, $\operatorname{diam}\left(\pi_{i}\left(F_{\sigma}\right)\right) \leq 1 / 2^{k}$. Since $\pi_{i}\left(y_{f}\right) \in \operatorname{cl}_{X}\left(\pi_{i}\left(F_{\sigma}\right)\right)$, we have $\pi_{i}\left(F_{\sigma}\right) \subset U_{i}$.

Case 2. $\phi(f \mid i)=0$.

Since $\pi_{i}\left(F_{\sigma}\right)$ is a singleton, $\pi_{i}\left(F_{\sigma}\right)=\left\{\pi_{i}\left(y_{f}\right)\right\}$, so $\pi_{i}\left(F_{\sigma}\right) \subset U_{i}$.

Thus, we have $\pi_{i}\left(F_{\sigma}\right) \subset U_{i}$ for all $i<n$, whence $F_{\sigma} \subset U$. Thus, the intersection of any neighborhood of $y_{f}$ with $F$ is uncountable, so $y_{f}$ is a limit point for $F$.

(1.4) Corollary. If $A$ is a set in a Polish space $X$ such that $|X \backslash A|<\mathfrak{c}$, then $A$ is holding. 
This provides a positive answer to Question 3 in [OY]. The following statement gives answers to Questions 1 and 2 in $[\mathrm{OY}]$.

(1.5) Theorem. Let $X$ be an uncountable Polish space. Then there exists a family of pairwise disjoint holding subsets $\left\{A_{\alpha}: \alpha<\mathfrak{c}\right\}$ of $X$.

Proof. Let $\left\{\Psi_{\beta}: \beta<\mathfrak{c}\right\}$ be a (transfinite) sequence of countable families of continuous finite-to-one functions from $\mathbf{C}=2^{\omega}$ to $X$ in which every family appears $\mathfrak{c}$ times. For $\beta<\mathfrak{c}, P \subset X$ and $Q \subset \mathbf{C}$, denote $\Psi_{\beta}(Q)=\bigcup\left\{\psi(Q): \psi \in \Psi_{\beta}\right\}$ and $\Psi_{\beta}^{-1}(P)=\bigcup\left\{\psi^{-1}(P): \psi \in \Psi_{\beta}\right\}$. Note that always $\left|\Psi_{\beta}(Q)\right| \leq \omega \cdot|Q|$ and $\left|\Psi_{\beta}^{-1}(P)\right| \leq \omega \cdot|P|$ (because the mappings in $\Psi_{\beta}$ are finite-to-one).

Let $\Gamma$ be the set of all pairs of ordinals $(\beta, \alpha)$ with $\alpha \leq \beta<\mathfrak{c}$; the lexicographic order $\prec$ is a well-ordering on $\Gamma$. Note that all initial segments in $(\Gamma, \prec)$ have cardinality $<\mathfrak{c}$. Let us construct by induction on $(\beta, \alpha)$ sequences $A_{\alpha}^{\beta}$ of subsets of $X$ and $x_{\alpha}^{\beta}$ of points of $\mathbf{C}$, so that for all $(\beta, \alpha) \in \Gamma$,

(1) $A_{\alpha}^{\beta}=\Psi_{\beta}\left(\left\{x_{\alpha}^{\beta}\right\}\right)$, and

(2) $x_{\alpha}^{\beta} \in \mathbf{C} \backslash \bigcup\left\{\Psi_{\beta}^{-1}\left(A_{\gamma}^{\delta}\right):(\delta, \gamma) \in \Gamma,(\delta, \gamma) \prec(\beta, \alpha)\right\}$.

Thus, at the step $(\beta, \alpha)$ we pick a point $x_{\alpha}^{\beta}$ in $\mathbf{C} \backslash \bigcup\left\{\Psi_{\beta}^{-1}\left(A_{\gamma}^{\delta}\right):(\delta, \gamma) \prec(\beta, \alpha)\right\}$ and put $A_{\alpha}^{\beta}=\Psi_{\beta}\left(\left\{x_{\alpha}^{\beta}\right\}\right.$ ) (the choice of $x_{\alpha}^{\beta}$ is possible, because the cardinality of the union does not exceed $\omega \cdot|\beta|<\mathfrak{c}$ ). Clearly, $A_{\alpha_{1}}^{\beta_{1}} \cap A_{\alpha_{2}}^{\beta_{2}}=\emptyset$ whenever $\left(\beta_{1}, \alpha_{1}\right) \neq\left(\beta_{2}, \alpha_{2}\right)$. Since every countable family $\Psi$ of finite-to-one continuous functions from $\mathbf{C}$ to $X$ occurs in the sequence $\mathfrak{c}$ times, for any $\alpha<\mathfrak{c}$ there is $\beta<\mathfrak{c}$ such that $\Psi=\Psi_{\beta}$ and $\alpha \leq \beta$, so by (1), $x_{\alpha}^{\beta}$ is in $\bigcap\left\{\psi^{-1}\left(A_{\alpha}^{\beta}\right): \psi \in \Psi\right\}$. Hence, the sets $A_{\alpha}=\bigcup\left\{A_{\alpha}^{\beta}: \alpha \leq \beta<\mathfrak{c}\right\}$ are disjoint holding subsets of $X$.

(1.6) Corollary. If $X$ is an uncountable Polish space, then there is a holding set $A \subset X$ such that $|X \backslash A|=\mathfrak{c}$.

We call a space analytic if it is a continuous image of $\omega^{\omega}[\mathrm{RJ}]$.

(1.7) Corollary. Every uncountable analytic space $X$ contains a holding set $A$ with $|X \backslash A|=\mathfrak{c}$.

Proof. Let $X$ be an uncountable analytic space. Then $X$ contains a homeomorphic copy $F$ of the Cantor cube $\mathbf{C}$ (this follows, for example, from Arhangel'skiü's generalization of the Hurewicz theorem, [Arh3]). By Corollary 1.6, there is a set $A_{0} \subset F$ that is holding in $F$ with $\left|F \backslash A_{0}\right|=\mathfrak{c}$. Put $A=A_{0} \cup(X \backslash F)$. Then $X \backslash A=F \backslash A_{0}$ has cardinality $\mathfrak{c}$, and we only need to check that $\left(X_{(A)}\right)^{\omega}$ is Lindelöf. We have $X_{(A)}=(X \backslash F) \cup F_{\left(A_{0}\right)}$, and $X \backslash F$ is a Borel set in $X$, hence analytic. Therefore, the next lemma ends the proof.

(1.8) Lemma. If $Y=Z \cup T$ where $Z^{\omega}$ is Lindelof and $T$ is analytic, then $Y^{\omega}$ is Lindelöf.

Proof of Lemma 1.8. Clearly, $Y$ is a continuous image of the disjoint union $Z \oplus \omega^{\omega}$, so it suffices to prove that $\left(Z \oplus \omega^{\omega}\right)^{\omega}$ is Lindelöf. The latter space is homeomorphic to a closed subspace of $(Z \oplus \omega)^{\omega}$, so we need to prove that this power is Lindelöf.

Consider two cases.

Case 1. $Z$ is compact. Then $Z \oplus \omega$ is $\sigma$-compact, so its countable power is Lindelöf.

Case 2. $Z$ is not compact. Then $Z$ is not countably compact, and there is a perfect mapping $p$ of $Z \oplus \omega$ onto $Z$. The countable power of $p$ is a perfect mapping of 
$(Z \oplus \omega)^{\omega}$ onto $Z^{\omega}$ [Eng, 3.7.6], so from the Lindelöf property of $Z^{\omega}$ follows the Lindelöf property of $(Z \oplus \omega)^{\omega}$ [Eng, 3.8.8]. The lemma is proved.

(1.9) Remark. As was observed by the referee, an uncountable metrizable $Q$-space cannot have holding sets with uncountable complement, so the existence of uncountable second-countable spaces in which every holding set has countable complement is consistent with ZFC. It is not clear whether such spaces can be constructed in ZFC.

(1.10) Remark. It is easy to see that a countable set $A$ cannot be holding in an uncountable first-countable space $X$. Indeed, if $A$ is a holding set, then it follows from Lemma 1.8 that $X_{(A)} \times \omega^{\omega}$ is Lindelöf; on the other hand, it is easy to prove that any $F_{\sigma \delta}$-set in a space $Z$ is the projection of a closed set in $Z \times \omega^{\omega}$. If $X$ is first countable and $A$ is a countable subset of $X$, then $X \backslash A$ is an uncountable discrete $F_{\sigma \delta}$-set in $X_{(A)}$, so $X_{(A)} \times \omega^{\omega}$ is not Lindelöf.

The cardinality of a holding set in an uncountable analytic space cannot be less than $\mathfrak{c}$. Indeed, every uncountable analytic space contains $\mathfrak{c}$ disjoint copies of the Cantor cube, and a holding set must meet each of these copies. Moreover, the following statement holds.

(1.11) Proposition. If $X$ is a Polish space, $A$ a holding set in $X$ and $B$ a subset of $X$ with $|B|<\mathfrak{c}$, then $A \backslash B$ is a holding set.

Proof. For any countable family $\Psi$ of continuous finite-to-one functions from $\mathbf{C}$ to $X$, since every Cantor set contains $\mathfrak{c}$ disjoint copies of itself, the intersection $\bigcap\left\{f^{-1}(A): f \in \Psi\right\}$ has cardinality $\mathfrak{c}$. Now if $|B|<\mathfrak{c}$, then the intersection $\bigcap\left\{f^{-1}(A \backslash B): f \in \Psi\right\}$ is nonempty. By Theorem 1.1, $A \backslash B$ is holding.

Theorem 1.1 has a "finite powers" version as follows.

(1.12) Theorem. Suppose $X$ is a Polish space, $A$ a subset of $X$, and $n$ a natural. Then the following conditions are equivalent:

(1) The space $\left(X_{(A)}\right)^{n}$ is Lindelöf,

(2) $\bigcap\left\{f^{-1}(A): f \in \Psi\right\} \neq \emptyset$ for any family $\Psi$ of $n$ finite-to-one continuous mappings of the Cantor cube $\mathbf{C}=2^{\omega}$ to $X$.

(3) $\bigcap\left\{f^{-1}(A): f \in \Psi\right\} \neq \emptyset$ for any family $\Psi$ of $n$ one-to-one continuous mappings of the Cantor cube $\mathbf{C}=2^{\omega}$ to $X$.

Proof. The implication $(1) \Rightarrow(2)$ is proved in exactly the same way as $(1) \Rightarrow(2)$ in Theorem 1.1 , and $(2) \Rightarrow(3)$ is trivial. We will now prove $(3) \Rightarrow(1)$ by induction on $n$.

Suppose $(3) \Rightarrow(1)$ is already proved for all $n<m$; assume for contradiction that (3) holds for $n=m$, and $Y^{m}$, where $Y=X_{(A)}$, is not Lindelöf.

As we have already mentioned in the proof of Theorem 1.1, from Baturov's theorem [Bat] follows that $Y^{m}$ has an uncountable closed discrete set $F$. Without loss of generality, $F$ has no isolated points in the topology of $X^{m}$ (formally, $i_{A I}^{m}(F)$ has no isolated points; we omit these obvious and cumbersome formulations and assume that we have two topologies on the set $X^{m}$, the Polish topology and that of $\left.Y^{m}\right)$.

Recall that $F$ is called $m$-countable [Pr1] if for any uncountable subset $F_{1}$ of $F$ there are $i<m$ and points $x_{1}, x_{2} \in F_{1}$ with $\pi_{i}\left(x_{1}\right)=\pi_{i}\left(x_{2}\right)$. 
Let us consider two possible cases.

Case 1. F is m-countable.

Then there are an uncountable $F_{1} \subset F$ and $i_{0}<m$ with $\left|\pi_{i_{0}}\left(F_{1}\right)\right|=1$ (see Lemma 1 in $[\operatorname{Pr} 1])$. The projection $Y^{m} \rightarrow Y^{m \backslash\left\{i_{0}\right\}}$ takes $F_{1}$ to a closed, discrete uncountable set in $Y^{m \backslash\left\{i_{0}\right\}}=Y^{m-1}$, in contradiction with the inductive assumption.

Case 2. F is m-uncountable.

Let $B$ be the closure of $F$ in the topology of $X^{m}$. By Theorem 1 in $[\operatorname{Pr} 1]$ $((\mathrm{i}) \Rightarrow(\mathrm{iv}))$, there is a family of one-to-one continuous functions $\left\{h_{0}, \ldots, h_{m-1}\right\}$ from C to $X$ such that $\left(h_{0}(c), \ldots, h_{m-1}(c)\right) \in B$ for all $c \in C$. By (3), there is a point $c_{0} \in C$ such that $h_{i}\left(c_{0}\right) \in A$ for $i<m$. The spaces $X^{m}$ and $Y^{m}$ have the same topology at the point $\left(h_{0}\left(c_{0}\right), \ldots, h_{m-1}\left(c_{0}\right)\right) \in A^{m}$, so this point must be a limit point for $F$ in $Y^{m}$, a contradiction with $F$ being closed and discrete.

(1.13) Theorem. For any $n \leq \omega$ and an uncountable completely metrizable space $X$, there exists a family $\left\{A_{\alpha}: \alpha<\mathfrak{c}\right\}$ of pairwise disjoint subsets of $X$ such that the spaces $\left(X_{\left(A_{\alpha}\right)}\right)^{m}$ are Lindelöf for all $m<n$ and $\alpha<\mathfrak{c}$, but the spaces $\left(X_{\left(A_{\alpha}\right)}\right)^{n}$ are not Lindelöf for any $\alpha<\mathfrak{c}$.

Proof. Fix a family $\Phi=\left\{g_{i}: i<n\right\}$ of continuous one-to-one functions from $\mathbf{C}$ to $X$ so that $g_{i}(\mathbf{C}) \cap g_{j}(\mathbf{C})=\emptyset$ whenever $i \neq j, i, j<n$ (this is possible, because every uncountable Polish space contains a homeomorphic copy of $\mathbf{C} \approx \mathbf{C} \times \mathbf{C}$ ). Let $\left\{\Psi_{\beta}: \beta<\mathfrak{c}\right\}$ be a sequence of all families of cardinality $<n$ of finite-to-one continuous functions from $\mathbf{C}$ to $X$ in which every family appears $\mathfrak{c}$ times.

As in the proof of Theorem 1.5, if $\Psi$ is a family of function from $\mathbf{C}$ to $X, P \subset \mathbf{C}$ and $Q \subset X$, we denote by $\Psi(P)$ the set $\bigcup\{f(P): f \in \Psi\}$, and by $\Psi^{-1}(Q)$, $\bigcup\left\{f^{-1}(Q): f \in \Psi\right\}$.

Put $\Gamma=\{(\beta, \alpha) \in \mathfrak{c} \times \mathfrak{c}: \alpha \leq \beta\}$; the lexicographic order $\prec$ is a well-ordering on $\Gamma$ whose all initial segments have cardinality $<\mathfrak{c}$. Similarly to the proof of Theorem 1.5, we may construct by transfinite induction on $(\beta, \alpha) \in \Gamma$ sequences $A_{\alpha}^{\beta}$ of subsets of $X$ and $x_{\alpha}^{\beta}$ of points of $\mathbf{C}$, so that for all $(\beta, \alpha) \in \Gamma$,

(1) $A_{\alpha}^{\beta}=\Psi_{\beta}\left(\left\{x_{\alpha}^{\beta}\right\}\right)$, and

(2) $x_{\alpha}^{\beta} \in \mathbf{C} \backslash \bigcup\left\{\Psi_{\beta}^{-1} \Phi \Phi^{-1}\left(A_{\alpha^{\prime}}^{\beta^{\prime}}\right):\left(\beta^{\prime}, \alpha^{\prime}\right) \prec(\beta, \alpha)\right\}$.

The sets $A_{\alpha}=\bigcup\left\{A_{\alpha}^{\beta}: \beta<\mathfrak{c}, \beta \geq \alpha\right\}$ are disjoint, and the spaces $\left(X_{\left(A_{\alpha}\right)}\right)^{m}$ are Lindelöf for all $m<n$ by Theorem 1.12 (Theorem 1.1 if $n=\omega$ ).

Let us now fix an $\alpha<\mathfrak{c}$ and check that $\bigcap\left\{g_{i}^{-1}\left(A_{\alpha}\right): i<n\right\}=\emptyset$, so condition (2) of Theorem 1.12 (of Theorem 1.1 if $n=\omega$ ) fails, and $\left(I_{\left(A_{\alpha}\right)}\right)^{n}$ is not Lindelöf.

Suppose for contradiction that $x \in \bigcap\left\{g_{i}^{-1}\left(A_{\alpha}\right): i<n\right\}$. Then $g_{i}(x) \in A_{\alpha}$, and there are $\beta_{i}<\mathfrak{c}, i<n$ such that $g_{i}(x) \in A_{\alpha}^{\beta_{i}}=\Psi_{\beta_{i}}\left(\left\{x_{\alpha}^{\beta_{i}}\right\}\right)$. Since the images of $C$ under $g_{i}, i<n$ are disjoint, we have $g_{i}(x) \neq g_{j}(x)$ whenever $i \neq j$. Furthermore, $\left|\Psi_{\beta}\left(x_{\alpha}^{\beta}\right)\right|<n$ for all $(\beta, \alpha) \in \Gamma$ (because $\left.\left|\Psi_{\beta}\right|<n\right)$; it follows that $\left(\beta_{j}, \alpha\right) \prec\left(\beta_{k}, \alpha\right)$ for some $j, k<n$. But then $x_{\alpha}^{\beta_{k}} \in \Psi_{\beta_{k}}^{-1} \Phi(x)$, and $x \in \Phi^{-1}\left(A_{\alpha}^{\beta_{j}}\right)$, so $x_{\alpha}^{\beta_{k}} \in \Psi_{\beta_{k}}^{-1} \Phi \Phi^{-1}\left(A_{\alpha}^{\beta_{j}}\right)$, in contradiction with property (2) of the construction.

(1.14) Remark. The existence of a single set $A \subset \mathbb{R}$ as in Theorem 1.13 (for any $n \leq \omega)$ may also be derived from Theorems 1 and 2 in [La]. 


\section{EXAMPLES RELATED TO FUNCTION SPACES}

It is well known [Zen], [Vel] that all finite powers of a space $X$ are hereditarily separable if and only if all finite powers of $C_{p}(X)$ are hereditarily Lindelöf. On the other hand, if $X$ is separable, and $C_{p}(X)$ is a Lindelöf $\Sigma$-space, then $X$ has countable network (this follows from II.6.9 and II.6.21 in [Arh1]). In [Pol] it is proved that if $X$ is a separable, scattered compact space whose $n$th derivative space is empty for some $\alpha<\omega_{1}$ and $C_{p}(X)$ is Lindelöf, then $X$ is metrizable (recall that the $\alpha$ th derivative space $X^{(\alpha)}$ of a space $X$ is defined inductively by the rules $X^{(0)}=X$, $X^{(\alpha+1)}=\left(X^{(\alpha)}\right)^{\prime}$ and $X^{(\alpha)}=\bigcap\left\{X^{(\beta)}: \beta<\alpha\right\}$ if $\alpha$ is a limit ordinal; here $Z^{\prime}$ denotes the set of all nonisolated points of $Z$ ). Reznichenko proved (see [Arh1]) that if MA $+\neg \mathrm{CH}$ holds, then every separable compact space $X$ with Lindelöf $C_{p}(X)$ is metrizable. Probably, this, together with several related results [AU], [Sip] led Arhangel'skiu to the question [Arh1]: Suppose $X$ is a separable compact space, and $C_{p}(X)$ is Lindelöf. Must $X$ be hereditarily separable?

The following example shows that the above statements related to separable compact spaces are not true for separable, scattered, $\sigma$-compact spaces. An example of a separable, $\sigma$-compact space $X$ such that $C_{p}(X)$ is Lindelöf but $X$ contains an uncountable discrete set has been obtained in $[\mathrm{OY}]$; the example depended on the existence of a holding set with uncountable complement in $I$, which was shown in [OY] under Martin's Axiom. Now we have a holding subset of $I$ with an uncountable complement in ZFC, so the example can be constructed in ZFC. We modify slightly the construction of $[\mathrm{OY}]$ to obtain a scattered space. Let us first recall two useful notions [Arh1].

A subset $S$ of $C_{p}(X)$ generates the topology of $X$ (or is a generating set of functions ) if the sets $f^{-1}(U)$, where $f \in S$ and $U$ is an open set in $\mathbb{R}$, form an open subbase for the topology of $X$. Thus, a set of continuous functions $S$ is generating if and only if for any point $x \in X$ and a closed set $F$ in $X$ with $x \notin F$ there are $n \in \omega$, functions $f_{1}, \ldots, f_{n} \in S$ and open subsets of $\mathbb{R}, U_{1}, \ldots, U_{n}$ such that $x \in \bigcap_{i=1}^{n} f_{i}^{-1}\left(U_{i}\right) \subset X \backslash F$. Obviously, a set of functions on a compact space $X$ is generating if and only if it separates points of $X$; in particular, if $X$ is compact and $S$ is a dense subset of $C_{p}(X)$, then $S$ is generating.

Let $Y$ be a subspace of $C_{p}(X)$. The evaluation mapping $\Phi_{X Y}: X \rightarrow C_{p}(Y)$ is defined by the rule:

$$
\Phi_{X Y}(x)(f)=f(x) \quad \text { for all } x \in X \text { and } f \in Y .
$$

The evaluation mapping coincides with the diagonal product $\Delta\{f: f \in Y\}: X \rightarrow$ $\mathbb{R}^{Y}$ (see [Eng, 2.3]); it is easy to check that $\Phi_{X Y}$ embeds $X$ in $C_{p}(Y)$ if and only if the set of functions $Y$ generates the topology of $X$.

(2.1) Theorem. There exists a separable, $\sigma$-compact space $X$ such that $X^{(3)}=\emptyset$, $C_{p}(X)^{\omega}$ is Lindelöf, and $X$ has an uncountable discrete subspace.

Proof. Let $A$ be a holding set in $I$ with $|I \backslash A|=\mathfrak{c}$. For each $r \in I$, denote by $\chi_{r}$ the characteristic function of the one-point set $\{r\}$, and consider the set of functions $Z_{A}=\left\{\chi_{r}: r \in I \backslash A\right\} \cup\{0\}$ where 0 is the zero function on $I$; clearly, $Z_{A} \subset C_{p}\left(I_{(A)}\right)$. Furthermore, $Z_{A}$ is a compact space with one nonisolated point, because any neighborhood of 0 in $\mathbb{R}^{I}$ contains all but finitely many points of $Z_{A}$.

Fix a dense countable subset $S$ of $C_{p}(I)$ (such a set exists, because $C_{p}(I)$ has countable network; see [Arh1]), and put $Y_{A}=S \cup Z_{A}$ (endowed with the topology 
of subspace of $\mathbb{R}^{I}$ ). Note that $Y_{A}$ generates the topology of $I_{(A)}$. Finally, put $X=X_{A}=\left(Y_{A}\right)_{\left(Z_{A}\right)}$ (so we make the points of $S$ isolated, with the possible exception of 0 ). Clearly, $X$ is a separable, $\sigma$-compact space and $X^{(3)}=\emptyset$, so we only need to check that $C_{p}(X)^{\omega}$ is Lindelöf.

Consider the evaluation mapping $\Phi_{I_{(A)} Y_{A}}: I_{(A)} \rightarrow C_{p}\left(Y_{A}\right)$ and put $M_{A}=$ $\Phi_{I_{(A)} Y_{A}}\left(I_{(A)}\right) \cup\left\{\chi_{s}: s \in S\right\} \cup\{0\} \subset \mathbb{R}^{Y_{A}}$. The set $M_{A}$ is a union of the space $\Phi_{I_{(A)} Y_{A}}\left(I_{(A)}\right)$, which is homeomorphic to $I_{(A)}$ because $Y_{A}$ generates the topology of $I_{(A)}$, and a compact set $K=\left\{\chi_{s}: s \in S\right\} \cup\{0\}$. By Lemma 1.8, $M_{A}^{\omega}$ is Lindelöf.

The topology on the set $Y_{A}$ generated by the set of functions $M_{A}$ is that of $X_{A}$; thus, $M_{A} \subset C_{p}\left(X_{A}\right)$, and the evaluation mapping $\Phi_{X_{A} M_{A}}$ embeds $X_{A}$ in $C_{p}\left(M_{A}\right)$.

Let $M_{A}^{+}$be the disjoint union of $M_{A}$ and a singleton; by Lemma 1.8, $\left(M_{A}^{+}\right)^{\omega}$ is Lindelöf. Furthermore, $C_{p}\left(M_{A}^{+}\right)=C_{p}\left(M_{A}\right) \times \mathbb{R}$; since $X_{A}$ embeds in $C_{p}\left(M_{A}\right)$, $X_{A} \times \omega$ admits an embedding in $C_{p}\left(M_{A}^{+}\right)$. By Corollary 2.8 in [Ok1], $C_{p}(X)^{\omega}=$ $C_{p}\left(X_{A}\right)^{\omega}=C_{p}\left(X_{A} \times \omega\right)$ is Lindelöf, and the proof is complete.

One of the most intriguing questions in the theory of function spaces in pointwise topology is whether the Lindelöf property of $C_{p}(X)$ implies the Lindelöf property of $C_{p}(X) \times C_{p}(X)$, in particular, when $X$ is compact (see [Arh2]). The question of the multiplicativity of the Lindelöf property in case where the spaces are different also makes sense; the strongest so far was an example, in a specially constructed model of ZFC, of two Lindelöf spaces $X_{1}$ and $X_{2}$, each with one nonisolated point, such that $C_{p}\left(X_{1}\right)$ and $C_{p}\left(X_{2}\right)$ are Lindelöf, but $C_{p}\left(X_{1}\right) \times C_{p}\left(X_{2}\right)$ is not [LM].

(2.2) Theorem. There exist separable, scattered, $\sigma$-compact spaces $X_{1}$ and $X_{2}$ such that $C_{p}\left(X_{1}\right)^{\omega}$ and $C_{p}\left(X_{2}\right)^{\omega}$ are Lindelöf, but $C_{p}\left(X_{1}\right) \times C_{p}\left(X_{2}\right)$ is not.

Proof. Let $A$ and $B$ be disjoint holding subsets of $I$. Construct the space $X_{A}$ as in the proof of Theorem 2.1, and let $X_{B}$ be the space constructed in the same way using the set $B$ instead of $A$. The same argument as in the proof of Theorem 2.1 shows that $C_{p}\left(X_{A}\right)^{\omega}$ and $C_{p}\left(X_{B}\right)^{\omega}$ are Lindelöf. Let us check that the product $C_{p}\left(X_{A}\right) \times C_{p}\left(X_{B}\right)$ contains a closed, discrete subspace of cardinality $\mathfrak{c}$.

The diagonal is a closed discrete set in $I_{(A)} \times I_{(B)}$ of cardinality $\mathfrak{c}$, so $I_{(A)} \times I_{(B)}$ is not Lindelöf. Therefore, it suffices to check that $I_{(A)}$ and $I_{(B)}$ are homeomorphic to closed subsets of $C_{p}\left(X_{A}\right)$ and $C_{p}\left(X_{B}\right)$. Of course, the proofs are similar, so we will check this for $C_{p}\left(X_{A}\right)$.

Let $S_{0}=i_{X_{A} Y_{A}}^{-1}(S)$ be the dense, countable, discrete set in $X_{A}=\left(Y_{A}\right)_{\left(Z_{A}\right)}$ (we keep the notation as in the proof of Theorem 2.1). The set $S$ generates the topology of $I$, so $\Phi_{I S}: I \rightarrow C_{p}(S) \subset C_{p}\left(S_{0}\right)=\mathbb{R}^{S}$ is an embedding. Since $I$ is compact, $\Phi_{I S}(I)$ is closed in $\mathbb{R}^{S}$.

Now let $\Phi_{I_{(A)} X_{A}}: I_{(A)} \rightarrow C_{p}\left(X_{A}\right)$ be the evaluation mapping and $R: C_{p}\left(X_{A}\right) \rightarrow$ $C_{p}\left(S_{0}\right)=\mathbb{R}^{S}$ the restriction mapping. Since $X_{A}$ generates the topology of $I_{(A)}$, the space $T=\Phi_{I_{(A)} X_{A}}\left(I_{(A)}\right)$ is homeomorphic to $I_{(A)}$, and since $S_{0}$ is dense in $X_{A}, R$ is (continuous) one-to-one. Furthermore, a simple verification shows that $R \circ \Phi_{I_{(A)} X_{A}}=\Phi_{I S} \circ i_{A I}$ (recall that $i_{A I}: I_{(A)} \rightarrow I_{(I)}=I$ is the natural bijection). Hence $T=R^{-1}\left(\Phi_{I S}(I)\right)$, so $T$ is closed in $C_{p}\left(X_{A}\right)$. The proof is complete.

It is easy to see that the diagonal in the product $X_{(A)} \times(X \backslash A)$ (the topology of the second factor is that of the subspace of $X$ ) is a closed, discrete set of cardinality $|X \backslash A|$. It follows that there is a set $A \subset I$ such that $\left(I_{(A)}\right)^{\omega}$ is Lindelöf, but $I_{(A)} \times(I \backslash A)$ is not. 
(2.3) Lemma. Let $Y$ be a separable, metrizable space. Then there is a countable space $M$ such that $Y$ is homeomorphic to a closed subspace of $C_{p}(M)$.

Proof. Let $K$ be a metrizable compactification of $Y$ and $\mathcal{B}$ a countable base for $K$. For any $U, V \in \mathcal{B}$ with $\operatorname{cl}(U) \subset V$ choose a continuous function $f_{U V}: K \rightarrow I$ so that $f(U) \subset\{1\}$ and $f(K \backslash V) \subset\{0\}$ and put $\mathcal{F}=\left\{f_{U V}: U, V \in \mathcal{B}, \operatorname{cl}(U) \subset\right.$ $V\} \cup\{0\}$. Clearly, the family of functions $\mathcal{F}$ is countable; endow it with the topology of subspace of $C_{p}(K)$.

Put $M=R(\mathcal{F})$ where $R: C_{p}(K) \rightarrow C_{p}(Y)$ is the restriction mapping. Since $Y$ is dense in $K$, the mapping $R_{0}=R \mid \mathcal{F}: \mathcal{F} \rightarrow M$ is a continuous bijection. It follows that the dual mapping $R_{0}^{*}: C_{p}(M) \rightarrow C_{p}(\mathcal{F})$ (defined by the rule $R_{0}^{*}(g)=g \circ R_{0}$ for all $g \in C_{p}(M)$; see [Arh1]) is an imbedding.

Clearly, $\mathcal{F}$ generates the topology of $K$ and $M$ generates the topology of $Y$, so the evaluation mappings $\Phi_{Y M}: Y \rightarrow C_{p}(M)$ and $\Phi_{K \mathcal{F}}: K \rightarrow C_{p}(\mathcal{F})$ are imbeddings. Let us check that $\Phi_{Y M}(Y)$ is closed in $C_{p}(M)$. The space $K$ is compact, so $\Phi_{K \mathcal{F}}(K)$ is closed in $C_{p}(\mathcal{F})$, and it is sufficient to check the equality $\Phi_{Y M}(Y)=R_{0}^{*-1}\left(\Phi_{K \mathcal{F}}(K)\right)$, or the equivalent equality $R_{0}^{*}\left(\Phi_{Y M}(Y)\right)=\Phi_{K \mathcal{F}}(K) \cap$ $R_{0}^{*}\left(C_{p}(M)\right)$.

Let $i: Y \rightarrow K$ be the imbedding. It is easy to check from the definitions of the evaluation mapping and the dual mapping that $R_{0}^{*} \circ \Phi_{Y M}=\Phi_{K \mathcal{F}} \circ i$. It follows that $R_{0}^{*}\left(\Phi_{Y M}(Y)\right) \subset \Phi_{K \mathcal{F}}(K) \cap R_{0}^{*}\left(C_{p}(M)\right)$.

Now suppose $g_{0} \in \Phi_{K \mathcal{F}}(K) \backslash R_{0}^{*}\left(\Phi_{Y M}(Y)\right)$; let us show that $g_{0} \notin R_{0}^{*}\left(C_{p}(M)\right)$. Suppose for contradiction that $g_{0}=R_{0}^{*}\left(h_{0}\right)$ for some $h_{0} \in C_{p}(M)$. Since $g_{0} \in$ $\Phi_{K \mathcal{F}}(K)$, we have $g_{0}=\Phi_{K \mathcal{F}}\left(x_{0}\right)$ for some $x_{0} \in K$, and from $g_{0} \notin R_{0}^{*}\left(\Phi_{Y M}(Y)\right)$ follows $x_{0} \notin Y$. Fix a sequence $\left\{U_{n}: n \in \omega\right\}$ of sets in $\mathcal{B}$ so that $\operatorname{cl}\left(U_{n+1}\right) \subset U_{n}$ and $\bigcap\left\{U_{n}: n \in \omega\right\}=\left\{x_{0}\right\}$ and put $f_{n}=f_{U_{n} U_{n+1}}, f_{n}^{\prime}=f_{n} \mid Y$ for all $n \in \omega$. Then the zero function is a limit point for the set of functions $F=\left\{f_{n}^{\prime}: n \in \omega\right\} \subset M$. Since $g_{0}=\Phi_{K \mathcal{F}}\left(x_{0}\right)$, we have $g_{0}(0)=0$ and $g_{0}\left(f_{n}\right)=f_{n}\left(x_{0}\right)=1$. By the definition of $R_{0}^{*}$, we have $h_{0}(0)=g_{0}(0)=0$ and $h_{0}\left(f_{n}^{\prime}\right)=g_{0}\left(f_{n}\right)=1$, so $h_{0}$ is discontinuous at 0 , a contradiction. The lemma is proved.

As an immediate consequence, we get the following statement.

(2.4) Theorem. There are a separable, scattered, $\sigma$-compact space $X$ and a countable space $M$ such that $C_{p}(X)^{\omega}$ is Lindelöf and $C_{p}(X) \times C_{p}(M)$ contains a closed discrete set of cardinality $\mathfrak{c}$.

(2.5) Remarks. 1. It is well known that if $X$ is separable metrizable and $X \backslash A$ is uncountable, then $X_{(A)} \times(X \backslash A)$ is nonnormal (the diagonal of $A$ and $A \times(X \backslash A)$ cannot be separated).

A similar argument shows that if $A$ and $B$ are disjoint subsets of $X, A$ is uncountable and $X_{(B)}$ Lindelöf, then $X_{(A)} \times X_{(B)}$ is not normal. Thus, the products in Theorems 2.2 and 2.4 are also nonnormal.

2. If $M$ is countable, then $C_{p}(M)$ is separable metrizable. Thus, Theorem 2.4 provides, in particular, an example of locally convex linear topological spaces, one of which is Lindelöf and the other second-countable, whose product is nonnormal and has a closed discrete subset of cardinality $\mathfrak{c}$.

In [Pol] an example of a separable scattered compact space $X$ such that $C_{p}(X, 2)^{n}$ is Lindelöf for any $n<\omega$, but $C_{p}(X, 2)^{\omega}$ is not Lindelöf is constructed under $\mathrm{CH}$. No such example is possible in ZFC: it is shown in [Ok2] that if $\mathrm{MA}+\neg \mathrm{CH}, X$ is 
separable compact, $Y \subset C_{p}(X)$ and all finite powers of $Y$ are Lindelöf, then $Y$ has countable network. Theorem 1.13 can be used to construct a $\sigma$-compact example:

(2.6) Theorem. There is a scattered, separable, zero-dimensional $\sigma$-compact space $X$ such that for any $n<\omega, C_{p}(X, 2)^{n}$ is Lindelöf, but $C_{p}(X, 2)^{\omega}$ is not Lindelöf.

Proof. Let $A$ be a subset of $I$ such that all finite powers of $I_{(A)}$ are Lindelöf, but the countable power is not Lindelöf (such a set exists by Theorem 1.13). As in the proof of Theorem 2.1, let $Z=\left\{\chi_{r}: r \in I \backslash A\right\} \cup\{0\}$, let $S$ be a dense countable set in $C_{p}(I), Y=S \cup Z$ and $X=Y_{(Z)}$. The space $X$ is $\sigma$-compact, scattered, zero-dimensional and separable; exactly the same argument as in the proof of Theorem 2.1 shows that $I_{(A)}$ is homeomorphic to a closed subset of $C_{p}(X)$. It follows that $C_{p}(X)^{\omega}$ is not Lindelöf.

Suppose $C_{p}(X, 2)^{\omega}$ is Lindelöf. Since $X$ is zero-dimensional, $C_{p}(X, 2)$ generates the topology of $X$, and the evaluation mapping imbeds $X$ in $C_{p}\left(C_{p}(X, 2)\right)$. Therefore, $X \times \omega$ is homeomorphic to a subspace of $C_{p}\left(C_{p}(X, 2)^{+}\right)=C_{p}\left(C_{p}(X, 2)\right) \times \mathbb{R}$ where $C_{p}(X, 2)^{+}$is the free sum of $C_{p}(X, 2)$ and a singleton. By Corollary 2.8 in [Ok1], $C_{p}(X \times \omega)=C_{p}(X)^{\omega}$ is Lindelöf, a contradiction.

Let us now check that all finite powers of $C_{p}(X, 2)^{n}$ are Lindelöf. We have $X \subset$ $C_{p}\left(M_{A}\right)$ where $M_{A}=\Phi_{I_{(A)}}\left(I_{(A)}\right) \cup\left\{\chi_{s}: s \in S\right\} \cup\{0\}$ is constructed exactly as in the proof of Theorem 2.1, and all finite powers of $M_{A}$ are Lindelöf. Furthermore, the set $Z=X^{\prime}$ is compact. Obviously, the property "all finite powers are Lindelöf" is inherited by closed subspaces, finite powers, continuous images, countable unions and products with compact spaces. Therefore, the Lindelöf property of all finite powers of $C_{p}(X, 2)$ follows from the next lemma.

(2.7) Lemma. Let $T$ be a space and $X \subset C_{p}(T)$. If the set $X^{\prime}$ of all nonisolated points of $X$ is compact, then $C_{p}(X, 2)$ is a countable union of continuous images of closed subsets of products of finite powers of $T$ with a compact space.

Proof of the lemma. Obviously, a function $\phi: X \rightarrow 2$ is continuous if and only if it is continuous at each point of $X^{\prime}$. Let us check that

$$
C_{p}(X, 2)=\bigcup_{m \in \omega} \bigcup_{n \in \omega} M(n, m)
$$

where

$$
\begin{aligned}
M(n, m)= & \bigcup_{\left(t_{0}, \ldots, t_{n-1}\right) \in T^{n}}\left\{\phi \in 2^{X}: \text { for any } f \in X^{\prime} \text { and } g \in X,\right. \\
& \text { if } \left.\left|f\left(t_{i}\right)-g\left(t_{i}\right)\right|<1 / m, i=0, \ldots, n-1, \text { then } \phi(f)=\phi(g)\right\} .
\end{aligned}
$$

Obviously, every function in the right-hand side is continuous. Conversely, if $\phi: X \rightarrow 2$ is continuous, then for every $f \in X^{\prime}$ there is a neighborhood of the form $U\left(f, l, t_{0}, \ldots, t_{k-1}\right)=\left\{g \in X:\left|f\left(t_{i}\right)-g\left(t_{i}\right)\right|<1 / l, i=0, \ldots, k-1\right\}$ such that $\phi(f)=\phi(g)$ for all $g$ in this neighborhood. The sets $U\left(f, 3 l, t_{0}, \ldots, t_{k-1}\right)$ form an open cover of the compact set $X^{\prime}$; fix a finite subcover $U\left(f_{0}, 3 l_{0}, t_{0}^{0}, \ldots, t_{k_{1}-1}^{0}\right)$, $\ldots, U\left(f_{N-1}, 3 l_{N-1}, t_{0}^{N-1}, \ldots, t_{k_{N}-1}^{N-1}\right)$. Put $n=k_{1}+\cdots+k_{N},\left(t_{0}, \ldots, t_{n-1}\right)=$ $\left(t_{0}^{0}, \ldots, t_{k_{N}-1}^{N-1}\right)$ and $m=\max \left(3 l_{0}, \ldots, 3 l_{N-1}\right) . \quad$ Now if $f \in X, g \in X^{\prime}$ and $\left|f\left(t_{i}\right)-g\left(t_{i}\right)\right|<1 / m$ for all $i<n$, then there is a $k<N$ such that $\left|f\left(t_{i}\right)-f_{k}\left(t_{i}\right)\right|<1 / m<1 / l_{k}$, for all $i<n$, so $\phi(f)=\phi\left(f_{k}\right)$. Furthermore, $\left|g\left(t_{i}\right)-f_{k}\left(t_{i}\right)\right| \leq\left|g\left(t_{i}\right)-f\left(t_{i}\right)\right|+\left|f\left(t_{i}\right)-f_{k}\left(t_{i}\right)\right|<1 / m+1 / m<1 / l_{k}$, so also $\phi(g)=$ $\phi\left(f_{k}\right)$. Thus, for every $\phi \in C_{p}(X, 2)$ we have found $m, n \in \omega$ and $\left(t_{0}, \ldots, t_{n-1}\right) \in T^{n}$ 
such that for any $f \in X^{\prime}$ and $g \in X$, if $\left|f\left(t_{0}\right)-g\left(t_{0}\right)\right|<1 / m, \ldots, \mid f\left(t_{n-1}\right)-$ $g\left(t_{n-1}\right) \mid<1 / m$, then $\phi(f)=\phi(g)$, so $\phi$ is in $M(n, m)$.

Let $S(n, m)$ be the subset of $2^{X} \times T^{n}$ defined by

$$
\begin{aligned}
& S(n, m)=\left\{\left(\phi, t_{0}, \ldots, t_{n-1}\right): \text { for any } f \in X^{\prime} \text { and } g \in X,\right. \\
& \left.\quad \text { if }\left|f\left(t_{i}\right)-g\left(t_{i}\right)\right|<1 / m, i=0, \ldots, n-1, \text { then } \phi(f)=\phi(g)\right\} .
\end{aligned}
$$

It is easy to see that $S(n, m)$ is closed in $2^{X} \times T^{n}$ and that $M(n, m)$ is a continuous image of $S(n, m)$ (under the projection of $2^{X} \times T^{n}$ to the first factor).

This proves the lemma and completes the proof of Theorem 2.6.

\section{ACKNOWLEDGEMENT}

The authors would like to thank the referee whose comments inspired several results in this paper and J. Zhu, S. Todorcevic and M. Sakai for useful discussion.

\section{REFERENCES}

[Arh1] A. V. Arhangel'skiu, Topological Function Spaces, Kluwer Acad. Publ., Dordrecht-BostonLondon, 1992. MR 92i:54022

[Arh2] A. V. Arhangel'skiı, Problems in $C_{p}$-theory, Open problems in Topology (J. van Mill and G. M. Reed, ed.), North-Holland, Amsterdam, 90, pp. 601-616. MR 92c:54001

[Arh3] A. V. Arhangel'skiı̌, Hurewicz spaces, analytic sets, and fan tightness of function spaces, Soviet Math. Dokl. 33 (1986), 396-399. MR 87i:54079

[AU] A. V. Arhangelskir and V. V. Uspenskiǔ, On the cardinality of Lindelöf subspaces of function spaces, Comment. Math. Univ. Carolinae 27 (4) (1986), 673-676. MR 88d:54003

[Bat] D. P. Baturov, On subspaces of function spaces, Vestnik Mos. Un-ta, Ser. 1, Matem., Mekhan. (1987), no. 4 (Russian); English Transl. in Moscow Univ. Math. Bull. 43 (1987) no. 4, 75-78. MR 89a:54018

[Eng] R. Engelking, General Topology, PWN, Warszawa, 1977. MR 58:18316b

[La] L. B. Lawrence, Lindelöf spaces concentrated on Bernstein subsets of the real line, Proc. Amer. Math. Soc. 114 (1) (1992), 211-215. MR 92c:54028

[LM] A. G. Leiderman and V. I. Malykhin, Nonpreservation of final compactness for the multiplication of spaces of type $C_{p}(X)$, Siberian Math. J. 29 (1988), 65-72. MR 89h:54019

[Ok1] O. G. Okunev, On Lindelöf $\Sigma$-spaces of continuous functions in the topology of pointwise convergence, Topology Appl. 49 (1993), 149-166. MR 94b:54055

[Ok2] O. Okunev, On Lindelöf sets of continuous functions, Topology Appl. 63 (1995), 91-96. CMP 95:11

[OY] O. G. Okunev and I. V. Yashchenko, On spaces of continuous functions on separable $\sigma$-compact spaces, Vestnik Mos. Un-ta, Ser. 1, Matem., Mekhan. (1993), no. 5, 75-78 (Russian); English Transl. in Moscow Univ. Math. Bull. 48 (1993).

[Pol] R. Pol, Concerning function spaces on separable compact spaces, Bull. Acad. Pol. Sci., Ser. Astr., Mat., Phys 25 (10) (1977), 993-997. MR 57:1414

[Pr1] T. C. Przymusiński, On the notion of n-cardinality, Proc. Amer. Math. Soc. 69 (2) (1978), 333-338. MR 58:10456

[Pr2] T. C. Przymusiński, Normality and paracompactness in finite and Cartesian products, Fund. Math. 105 (1980), 87-104. MR 80m:54010

[RJ] A. Rogers and E. Jayne (eds.), Analytic Sets, Academic Press, London, 1980. MR 80m:03063

[Sip] O. Sipacheva, Lindelöf subspaces of functional spaces for linearly ordered separable compact spaces, General Topology. Spaces and Mappings, Moscow University Publ., Moscow, 1989, pp. 143-148 (Russian). CMP 91:08; MR 91j:54001

[Vel] N. V. Velichko, On the weak topology of spaces of continuous functions, Matem. Zametki 30 (5) (1981), 703-712 (Russian). MR 83f:54006

[Zen] Ph. Zenor, Hereditary $\mathfrak{m}$-separability and hereditary $\mathfrak{m}$-Lindelöf property in product spaces and function spaces, Fund. Math. 106 (3) (1981), 175-180. MR 82a:54039 
Computer Science and Engineering Laboratory, The University of Aizu, Ikki machi, Aizu-Wakamatsu City, Fukushima 965, Japan

E-mail address: o-okunev@rsc.u-aizu.ac.jp

Current address: Facultad de Ciencias, Departamento de Matematicas, Ciudad Universitaria, Circuito Exterior, C. P. 04510, Mexico D. F., Mexico

E-mail address: oleg@lya.fciencias.unam.mx

Department of Mathematics, Faculty of Engineering, Yokohama National UniverSity, 156 Tokiwadai, Hodogaya, Yokohama 240, JaPan

E-mail address: tamano@math.sci.ynu.ac.jp 